\title{
Safety and Efficacy using second-generation cryoballoon in patients with atrial fibrillation with a Common Ostium of Inferior Pulmonary Veins
}

\author{
Hai-yang Xie ${ }^{1}$, Xiao-gang Guo ${ }^{2}$, Jian-du Yang ${ }^{2}$, Hui-qiang Wei $^{3}$, Yan-qiao Chen ${ }^{2}$, \\ Zhong-jing $\mathrm{CaO}^{2}$, qi $\mathrm{sun}^{4}$, and Jian $\mathrm{Ma}^{2}$ \\ ${ }^{1}$ State Key Laboratory of Cardiovascular Disease, Arrhythmia Center, Fuwai Hospital, \\ National Center for Cardiovascular Diseases, Chinese Academy of Medical Sciences and \\ Peking Union Medical College \\ ${ }^{2}$ Fuwai Hospital, National Center for Cardiovascular Diseases, Chinese Academy of Medical \\ Sciences and Peking Union Medical College, Beijing, China \\ ${ }^{3}$ Guangdong Provincial People's Hospital Guangdong Cardiovascular Institute \\ ${ }^{4}$ Chinese Academy of Medical Sciences and Peking Union Medical College
}

November 3, 2020

\begin{abstract}
Background: A common ostium of inferior pulmonary veins (COIPV) is a kind of unusual pulmonary veins variation. Limited data are available about the safety and efficacy using the second-generation cryoballoon $(\mathrm{CB})$ ablation in patients with atrial fibrillation with such an anatomical variation. Methods: A total of 10 (0.57\%) patients with a common ostium of inferior pulmonary veins from a consecutive series of 1751 consecutive patients with atrial fibrillation (AF) were included. Pulmonary vein isolation was performed using the second-generation CB. Results: PV isolation was successfully achieved in all PVs without the need of additional focal catheter procedures. CB could occlude and cryotherapy each inferior PV respectively. The mean number of freeze cycles of inferior PVs in each patient was $2.5 \pm 1.6$, and $2.1 \pm 0.9$, respectively. The mean time to isolation and nadir temperature were $54.3 \pm 22.1,53.2 \pm 22.2$ seconds and $-39.7 \pm 3.4^{\circ} \mathrm{C},-43.9 \pm 7.5^{\circ} \mathrm{C}$, respectively. No procedure-related complications occurred in any of the 10 patients. 8 of 10 patients $(80 \%)$ had no atrial arrhythmia recurrences without the use of antiarrhythmic drugs during a follow-up period of $24 \pm 13$ months. Conclusion: The incidence of a common ostium of inferior pulmonary veins is $0.57 \%$ in present study. PV isolation with this unusual anatomic variation using the second-generation $28-\mathrm{mm} \mathrm{CB}$ is effective and safe.
\end{abstract}

Safety and Efficacy Using Second-generation Cryoballoon in Patients with Atrial Fibrillation with A Common Ostium of Inferior Pulmonary Veins

Xie, Hai-yang, MD*; Guo, Xiao-gang, MD*; Yang , Jian-du, MD*; Wei, Hui-qiang, MD \#; Chen, Yan-qiao, MD*; Cao, Zhong-jing, MD*; Sun, Qi, MD*; Ma, Jian, MD*1

* State Key Laboratory of Cardiovascular Disease, Arrhythmia Center, Fuwai Hospital, National Center for Cardiovascular Diseases, Chinese Academy of Medical Sciences and Peking Union Medical College

\# Guangdong Provincial People's Hospital Guangdong Cardiovascular Institute

${ }^{1}$ Corresponding author: State Key Laboratory of Cardiovascular Disease, Arrhythmia Center, Fuwai Hospital, National Center for Cardiovascular Diseases, Chinese Academy of Medical Sciences and Peking 
Union Medical College, 100037 Beijing, China. E-mail: majian_fuwai@126.com. Phone: +8618811213672. Fax: +8601088398435 .

Funding information

This work was supported by a grant of National Natural Science Foundation of China (\#81670309).

Disclosures: None.

\section{Abstract}

Background: A common ostium of inferior pulmonary veins (COIPV) is a kind of unusual pulmonary veins variation. Limited data are available about the safety and efficacy using the second-generation cryoballoon (CB) ablation in patients with atrial fibrillation with such an anatomical variation.

Methods: A total of $10(0.57 \%)$ patients with a common ostium of inferior pulmonary veins from a consecutive series of 1751 consecutive patients with atrial fibrillation (AF) were included. Pulmonary vein isolation was performed using the second-generation CB.

Results: PV isolation was successfully achieved in all PVs without the need of additional focal catheter procedures. CB could occlude and cryotherapy each inferior PV respectively. The mean number of freeze cycles of inferior PVs in each patient was $2.5 \pm 1.6$, and $2.1 \pm 0.9$, respectively. The mean time to isolation and nadir temperature were $54.3 \pm 22.1,53.2 \pm 22.2$ seconds and $-39.7 \pm 3.4^{\circ} \mathrm{C},-43.9 \pm 7.5^{\circ} \mathrm{C}$, respectively. No procedure-related complications occurred in any of the 10 patients. 8 of 10 patients $(80 \%)$ had no atrial arrhythmia recurrences without the use of antiarrhythmic drugs during a follow-up period of $24 \pm 13$ months.

Conclusion: The incidence of a common ostium of inferior pulmonary veins is $0.57 \%$ in present study. PV isolation with this unusual anatomic variation using the second-generation 28-mm $C B$ is effective and safe.

Key words: catheter ablation, cryoballoon, atrial fibrillation, pulmonary vein, common ostium

\section{Introduction}

The two most common pulmonary veins (PVs) anomalies are the presence of a right middle pulmonary vein (PV) and common left trunk. However, a common ostium of inferior pulmonary veins (COIPV) is a kind of unusual variation. Radiofrequency ablation of atrial fibrillation (AF) in patients with that anomaly had been reported before ${ }^{[1]}$. Whereas, there was sporadic case report described previously in the context of PV isolation using by cryoballoon (CB) ablation ${ }^{[2]}$. Limited data are available about the safety and efficacy using the second-generation CB ablation in patients with atrial fibrillation with such an anatomical variation. Therefore, we aimed to explore the safety and efficacy of using second-generation CB in patients with AF with COIPV.

\section{METHODS}

\section{Study population}

In a total population of 1751 consecutive patients with AF who had undergone index ablation by second-generation CB between May 2016 and October 2019, 10 patients (0.57\%) with COIPV determined by multidetector computed tomography (MDCT) scan were included for final analysis. All patients provided written informed consent before the CB ablation. This study was approved by the Institutional Review Board.

Preprocedural management 
All antiarrhythmic drugs were discontinued at least five half-lives before ablation. Transthoracic echocardiography (TTE) was used to evaluate left atrial (LA) size and left ventricular ejection fraction (LVEF) in all patients. A transesophageal echocardiography before the procedure was to exclude the presence of intracavitary thrombi, and computed tomography (CT) was performed to analyze the detailed LA and PV anatomy.

CB ablation of PVs

The procedure was performed as previously described ${ }^{[3]}$. In brief, all cryoablation procedures were performed under deep sedation with continuous infusion of midazolam and fentanyl. After achieving LA access guided by fluoroscopy, a single 3-minute application was performed for each vein with the 28-mm CB ablation when optimal PV occlusion was achieved by a "proximal-seal" technique. Isolation of PVs was confirmed by using the inner lumen mapping catheter (Achieve, Medtronic, Minneapolis, MN). A bonus cryoablation was given for the PV when time to isolation (TTI) was greater than 60 seconds. And segmental freeze strategy would be performed if PV ostium was hard to get an optimal PV occlusion. If the balloon nadir temperature exceeded -60degC, the ablation would be terminated. During cryoablation of the right-sided PVs, high-output right phrenic stimulation $(1500 \mathrm{~ms} ; 20 \mathrm{~mA})$ was performed using a quadripolar catheter within the SVC. When loss of pacing capture occurred, the ablation was immediately terminated.

Follow-up

After discharge from the hospital, patients were scheduled for follow up visits at 1, 3, 6, and 12 months. For each visit, 24-hour Holter ECG recordings were performed to evaluate the atrial arrhythmias. 3 months after the index procedure was considered as the blanking period. Recurrence was defined as any documented atrial episodes with duration longer than 30 seconds.

Statistical Analysis

Continuous variables were described as mean +- standard deviation and categorical variables were expressed as percentages. Continuous variables were compared using the Student's t-test. A two-tailed value of $\mathrm{P}<0.05$ was considered statistically significant. All statistical analyses were performed using SPSS 19.0 (IBM Corp., Armonk, NY, USA).

Results

Baseline characteristics

A total of 10 in 1751 patients (9 males; 53 +- 8 years) undergoing AF ablation were determined to have a common ostium of inferior pulmonary veins via CT scan before the intervention. The average diameter of the common ostium was $30.4+-5 \mathrm{~mm}$, and the average diameter of the left atrium was $41.4+-2.8 \mathrm{~mm}$. No structural heart diseases were found in all patients. Patient characteristics are described in Table 1.

In 5 of the 10 patients, the esophaguses directly came in contact with Left inferior PV ostium, two cases directly came in contact with the junction part of the two inferior PVs, and in remaining three cases directly came in contact with the Left inferior PV branch but away from the ostium.

Procedural parameters of PV isolation

A total of $40 \mathrm{PVs}$ were targeted in the 10 patients. A 28-mm CB was applied in all patients and $\mathrm{PV}$ isolation was successfully achieved in all PVs without the need of touching up. A mean number of 8.8+-2.3 CB freezes were applied per patient and 2.8+-0.9, 1.4+-0.5, 2.5+-1.6, and 2.1+-0.9 for left superior PV, left inferior PV, right superior PV, and right inferior PV, 
respectively. Procedural and biophysical characteristics are displayed in Table 2. Although there was a common ostium of inferior PVs, CB could occlude and cryotherapy each inferior PV respectively. Compared to those without common ostium of inferior PVs, the higher nadir temperature was observed in patients with common ostium of inferior PVs (-39.7+-3.4degC vs -44.9+-5.5degC; $\mathrm{P}=0.029)$ and (-43.9+-7.5degC vs-50.7+-4.2degC; $\mathrm{P}=0.019)$ for left inferior PV and right inferior PV respectively. However, no significant difference in TTI (-54.3+22.1degC vs -44.1+-22.2degC; $P=0.197)$ and $(-47.9+-26.9 \operatorname{deg} C$ vs-55.3+-32.1deg $C ; P=0.508)$ respectively was found for patients.

\section{Follow up}

No procedure-related complications occurred in any of the 10 patients. 8 of 10 patients (80\%) had no atrial arrhythmia recurrences without the use of antiarrhythmic drugs during a followup period of 24+-13 months. A cardiac CT was performed in 3 patients during the follow up and no PV stenosis and atrio-esophageal fistula was detected. Furthermore, no patients had signs or symptoms of atrio-esophageal fistula, such as chest discomfort, nausea, vomiting, dysphagia, odynophagia, hematemesis, melena.

Discussion

\section{Major findings}

To the best of our knowledge, this is the first study that examines the safety and efficacy of using second-generation CB in patients with AF with COIPV. In the current study, we found that (1) COIPV was present in $0.57 \%$ of the patients included in the study. (2) CB ablation of the COIPV is feasible and safe, with acute isolation in $100 \%$ of these veins.

\section{COIPV cryoablation}

The two most common PVs anomalies are a right middle PV and common left trunk. In present study demonstrated a rare variation in the pattern of pulmonary veinous drainage. Rare studies previously described in the context of PV isolation. YU, R., et al ${ }^{[1]}$ had presented a series of PV isolation cases achieved by "tricircle" ablation strategy along the circumferential lines or by segmental PV ostium ablation. Up to $90 \%$ success rate could be acquired to isolate PV. Futhermore, Squara, F., et al. ${ }^{[4]}$ demonstrated another strategy performed isolation of the entire posterior wall with a single ring of ablation lesions to minimize lesions on the posterior wall, so as to minimize the risk of oesophageal injury. Radiofrequency catheter applied to such pattern of pulmonary veinous drainage had been proven available to achieve PV isolation. However, RF catheter tip hard to stabilize on the ridge between two inferior PVs so that had to adopt an unfamiliar strategy to cope with the difficulty. And extensive circumferential RF lines would be created. As the matter stands, the difficulty correspondingly increased many. Considering CB ablation can more conveniently create a circumferential lesion at the PV ostium. And the second-generation CB has been widely used to perform PV isolation due to its efficacy and safety ${ }^{[5,6]}$.However, whether this novel ablation technology could facilitate COIPV isolation and improve the safety of the procedure remains unknow. Defaye, P., et al. ${ }^{[2]}$ initially reported a case that PV isolation was successfully achieved by CB ablation without no complication. In the present study, we confirmed that COIPV can be isolated successfully by using second-generation CB without adverse events. Although inferior PVs shared a common ostium, optimal occlusion respectively using the 28-mm CB could be achieved and PV isolation was successfully achieved in all PVs without the need of additional focal catheter procedures. There was no significantly different operation compared to those without COIPV, but both inferior PVs grow more posteriorly and sheath ought to turn more posteriorly to send "Achieve" into PVs. After a mean follow-up period of about 24 months, $80 \%$ of patients had no recurrence of AF. Therefore, the electrical isolation of COIPV using the second-generation $\mathrm{CB}$ is feasible and effective. 


\section{Safety performance}

Anatomically, the left inferior PV ostium has the closest relationship to the esophagus. The potential for esophageal damage should always be a consideration. Although atrioesophageal fistula (AEF) formation associated with cryoballoon ablation is very rare, John, R. M., et al ${ }^{[7]}$ reported that 8 of $10 \mathrm{AEF}$ were recorded in relation to the left inferior PV. In this study, the esophaguses of $50 \%$ patients directly came in contact with left inferior PV ostium. What's more, two cases directly came in contact with the junction part of the two inferior PVs, where the freeze area may overlap. Previous study had demonstrated that the longer duration of balloon inflation was significantly associated with $\mathrm{AEF}^{[7]}$, and direct cooling with lower

temperature was considered to play the major role in $\mathrm{AEF}^{[8]}$. In our study, the higher nadir temperature and less during of CB freezes were observed, which may contribute to no AEF happened in all patients. Therefore, position the balloon proximally to avoid a precipitous drop in balloon temperatures and avoiding continuous ablating at the same PV are needed to protect esophagus.

Clinical implication

The use of second-generation CB is increasing worldwide and cryoablation procedure is no significantly different compared to those without COIPV. PV isolation with such unusual variation using second-generation $\mathrm{CB}$ might become a promising alternative ablation procedure. CT scan before the procedure can provide information for planning the ablation strategy and its adjacent anatomy.

Study limitations

The study was limited by its retrospective single-center design and relatively small sample size. Further randomized studies with a large sample are needed to confirm our findings.

Gastrointestinal endoscope was not used to confirm esophageal injury before and after procedure.

\section{CONCLUSION}

The incidence of a common ostium of inferior pulmonary veins is $0.57 \%$ in present study. $\mathrm{PV}$ isolation with this unusual anatomic variation using the second-generation 28-mm CB is feasible and safe.

Acknowledgments

1. YU R, DONG J, ZHANG Z, LIU X, KANG J, LONG D, et al.Characteristics in Image Integration System Guiding Catheter Ablation of Atrial Fibrillation with a Common Ostium of Inferior Pulmonary Veins . Pacing and Clinical Electrophysiology 2008; 31(1):93-98.

2. Defaye P, Kane A, Jacon P. An unusual connection of inferior pulmonary veins in the left atrium via a common ostium: a cardiac computed tomographic angiography discovery before cryoballoon pulmonary vein isolation in atrial fibrillation . Heart Asia 2011; 3(1):34.

3. Wei HQ, Guo XG, Zhou GB, Sun Q, Liu X, Yang JD, et al.Pulmonary vein isolation with realtime pulmonary vein potential recording using second-generation cryoballoon: Procedural and biophysical predictors of acute pulmonary vein reconnection .Pacing and clinical electrophysiology : PACE 2018; 41(1):14-21.

4. Squara F, Sureddi R, Marchlinski FE. Entire posterior wall isolation in an unusual case of common ostium between the right inferior and left inferior pulmonary veins . Europace 2014; $16(6): 839$. 
5. Metzner A, Reissmann B, Rausch P, Mathew S, Wohlmuth P, Tilz R, et al. One-year clinical outcome after pulmonary vein isolation using the second-generation 28-mm cryoballoon . Circ Arrhythm Electrophysiol 2014; 7(2):288-292.

6. Furnkranz A, Bordignon S, Dugo D, Perotta L, Gunawardene M, Schulte-Hahn B, et al. Improved 1-year clinical success rate of pulmonary vein isolation with the second-generation cryoballoon in patients with paroxysmal atrial fibrillation. J Cardiovasc Electrophysiol 2014; 25(8):840-844.

7. John RM, Kapur S, Ellenbogen KA, Koneru JN. Atrioesophageal fistula formation with cryoballoon ablation is most commonly related to the left inferior pulmonary vein . Heart Rhythm 2017; $14(2): 184-189$.

8. Miyazaki S, Nakamura H, Taniguchi H, Takagi T, Iwasawa J, Watanabe T, et al. Esophagus-Related Complications During Second-Generation Cryoballoon Ablation-Insight from Simultaneous Esophageal Temperature Monitoring from 2 Esophageal Probes . J Cardiovasc Electrophysiol2016; 27(9):1038-1044.

Table 1: Baseline characteristics

Note: Values are expressed as mean +- SD or as $\mathbf{n}(\%)$. Abbreviations: BMI, body mass index; LA, left atrial; LVEF, left ventricular ejection fraction.

\begin{tabular}{ll}
\hline Patients & $\mathrm{N}=10$ \\
\hline Age, y & $53 \pm 8$ \\
Male sex (\%) & $9(90)$ \\
BMI & $27.7 \pm 2.8$ \\
CHA2DS2-VASc (score) & $0.5 \pm 0.8$ \\
Hypertension, n (\%) & $2(20)$ \\
Diabetes mellitus, n (\%) & $\mathbf{0 ( 0 )}$ \\
Previous stroke, n (\%) & $\mathbf{0}(0)$ \\
LA diameter, mm & $41.4 \pm 2.8$ \\
LVEF (\%) & $63.1 \pm 1.9$ \\
\hline
\end{tabular}

Table 2: Procedural and biophysical characteristics

Note: Values are expressed as mean \pm SD or as $\mathbf{n}(\%)$. Abbreviation: LSPV, left superior pulmonary vein; LIPV, left inferior pulmonary vein; RSPV, right superior pulmonary vein; RIPV, right inferior pulmonary vein.

\begin{tabular}{ll}
\hline Mean total procedural time (minutes) & $\mathbf{5 2 . 9} \pm 7.4$ \\
\hline Mean fluoroscopy time (minutes) & $13.3 \pm 3.6$ \\
Mean number of applications, $\mathrm{n}$ & $8.8 \pm 2.3$ \\
LSPV & \\
Mean number of freezes, $\mathrm{n}$ & $\mathbf{2 . 8} \pm \mathbf{0 . 9}$ \\
Time to isolation (seconds) & $38.4 \pm 4.5$ \\
Temperature at isolation $\left({ }^{*} \mathrm{C}\right)$ & $\mathbf{- 3 6 . 7} \pm 3.8$ \\
Nadir temperature $\left({ }^{*} \mathrm{C}\right)$ & $-49.3 \pm 5.3$ \\
LIPV & \\
Mean number of freezes, $\mathrm{n}$ & $\mathbf{1 . 4} \pm \mathbf{0 . 5}$ \\
Time to isolation $($ seconds) & $\mathbf{5 4 . 3} \pm \mathbf{2 2 . 1}$ \\
Temperature at isolation $\left({ }^{*} \mathrm{C}\right)$ & $\mathbf{- 3 4 . 1} \pm 4.8$ \\
Nadir temperature $\left({ }^{*} \mathrm{C}\right)$ & $-39.7 \pm 3.4$ \\
RSPV &
\end{tabular}




\begin{tabular}{ll}
\hline Mean total procedural time (minutes) & $52.9 \pm 7.4$ \\
\hline Mean number of freezes, $\mathrm{n}$ & $2.5 \pm 1.6$ \\
Time to isolation (seconds) & $67.4 \pm 32.5$ \\
Temperature at isolation $\left({ }^{*} \mathrm{C}\right)$ & $-39.9 \pm 5.5$ \\
Nadir temperature $\left({ }^{*} \mathrm{C}\right)$ & $-51.1 \pm 3.0$ \\
RIPV & \\
Mean number of freezes, $\mathrm{n}$ & $\mathbf{2 . 1} \pm \mathbf{0 . 9}$ \\
Time to isolation $($ seconds) & $\mathbf{5 3 . 2} \pm \mathbf{2 2 . 2}$ \\
Temperature at isolation $\left({ }^{*} \mathrm{C}\right)$ & $-32.3 \pm 9.8$ \\
Nadir temperature $\left({ }^{*} \mathrm{C}\right)$ & $-43.9 \pm 7.5$ \\
\hline
\end{tabular}

Figure 1: 3-dimensional computer tomography images of left atrium and pulmonary veins. Abbreviation: PA, Posteroanterior; SUP, superior.

Figure 2: CB ablation of common ostiums of inferior pulmonary veins. A, The 28-mm CB was positioned at the ostium of LIPV and the PV potentials (arrow) were recorded in the Achieve catheter; B, The 28-mm CB was positioned at the ostium of RIPV, LIPV and the PV potentials (arrow) were recorded in the Achieve catheter. Abbreviation: CB, cryoballoon; CS , coronary sinus; RV, right ventricular; LAO, left anterior oblique ; RAO, right anterior oblique; LIPV, left inferior pulmonary vein; RIPV, right inferior pulmonary vein.
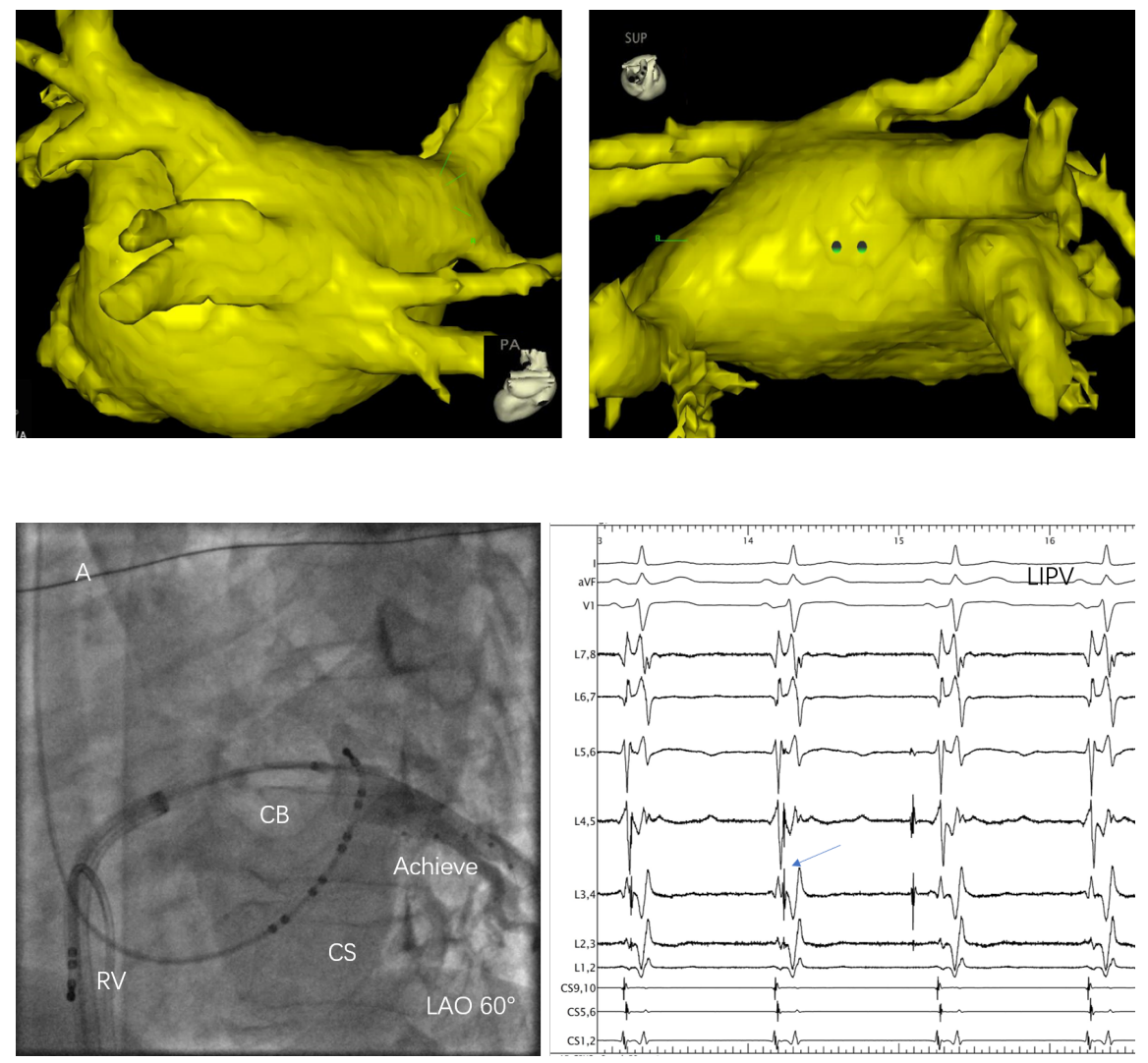

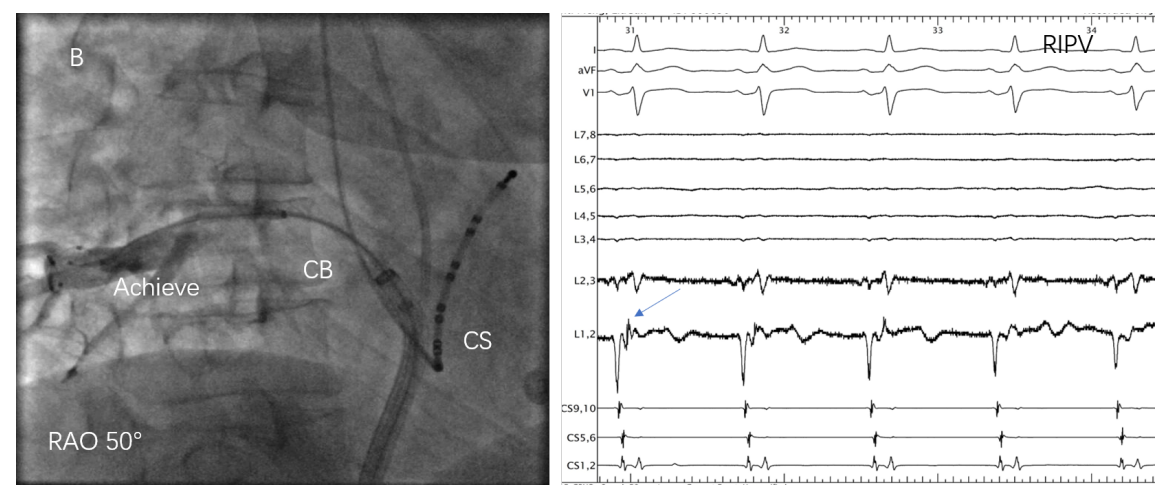